Chirurg 2013 $\cdot 84: 698$

DOI 10.1007/s00104-013-2571-3

Online publiziert: 19. Juni 2013

๑) Springer-Verlag Berlin Heidelberg 2013

H. Dralle

Medizinische Fakultät, Universitätsklinik für Allgemein-, Viszeral- und Gefäßchirurgie,

Universitätsklinikum Halle, Halle (Saale)

\title{
Ultraschalldiagnostik der Stimmlippenbeweglichkeit vor Schilddrüsenoperationen?
}

\section{Ergebnisse}

In der ersten Phase konnte bei 93 Patienten $(82 \%)$ ein Ultraschallbefund erhoben werden. Bei 21 Patienten war die SL-Beweglichkeit nicht ausreichend zu beurteilen. Zwei der 93 Patienten hatten eine auch laryngoskopisch bestätigte - Rekurrensparese (RP; 2,2\%).

In der zweiten Phase konnte bei 349 von insgesamt 415 Patienten (84\%) die SL-Beweglichkeit beurteilt werden. Bei einem der mit Ultraschall nicht ausreichend zu beurteilenden Patienten (1/66) wurde laryngoskopisch eine klinisch asymptomatische RP nachgewiesen. Vier der 349 sonographisch untersuchten $\mathrm{Pa}$ tienten $(1,5 \%)$ hatten eine abnorme SL-Beweglichkeit mit laryngoskopisch bestätigter RP. Bei keinem der 345 Patienten mit sonographisch normaler SL-Beweglichkeit wurde eine RP nachgewiesen. Insgesamt 56 der 345 Patienten hatten auffällige Stimmsymptome, ohne dass laryngoskopisch eine RP vorlag.

\section{Diskussion und Fazit}

\section{Methoden}

In 2 Untersuchungsphasen wurden insgesamt 463 Patienten vor Schilddrüsenund Nebenschilddrüsenoperationen hinsichtlich ihrer SL-Beweglichkeit sonographisch untersucht. In der ersten Phase erfolgte sowohl eine Laryngoskopie als auch eine Ultraschalluntersuchung $(\mathrm{n}=114)$. In der zweiten Phase wurde eine Laryngoskopie nur in den Fällen durchgeführt, bei denen im Ultraschall eine pathologische SL-Beweglichkeit festgestellt wurde, wenn die SL-Beweglichkeit nicht hinreichend zu beurteilen war oder wenn auffällige Stimmsymptome vorlagen.
Die Untersuchung bestätigt an einem umfangreichen Krankengut frühere Ergebnisse anderer Arbeitsgruppen [1], die ebenfalls zeigen konnten, dass die sonographische Diagnostik der SL-Beweglichkeit geeignet ist, bei ausreichender Beurteilbarkeit und normaler Beweglichkeit auf die aufwendigere und belastendere fiberoptische Laryngoskopie verzichten zu können. Der Anteil der Patienten, bei denen weiterhin eine Laryngoskopie erforderlich ist, liegt in der vorliegenden Untersuchung bei insgesamt $34 \%$ ( $16,5 \%$ wegen unzureichender sonographischer Beurteilbarkeit,
$1,5 \%$ wegen sonographisch abnormer SL-Beweglichkeit, 16,2\% wegen Stimmsymptomen). Das heißt, bei etwa zwei Drittel der Patienten konnte die SL-Beweglichkeit sonographisch ohne Qualitätsverlust (keine RP bei sonographisch eindeutig nachweisbarer intakter SL-Beweglichkeit) beurteilt werden.

Aufgrund der bislang zu dieser Methode vorliegenden Untersuchungen besteht hinreichend Grund, durch weitere Studien zu prüfen, ob die Ultraschalluntersuchung der SL-Beweglichkeit bei Schilddrüsen- und Nebenschilddrüsenoperationen die Routine-Larnygoskopie nicht nur präoperativ, sondern auch postoperativ ersetzen kann. Voraussetzung hierfür ist, dass keine auffälligen Stimmsymptome vorliegen und der UItraschall eindeutig eine normale SL-Beweglichkeit zeigt. Zur Abklärung laryngealer Ursachen von Stimmstörungen und Funktionsstörungen der Stimmlippen ist der Ultraschall aus methodischen Gründen nicht geeignet.

\section{Korrespondenzadresse}

Prof. Dr. Dr. h.c. H. Dralle

Medizinische Fakultät, Universitätsklinik für Allgemein-, Viszeral- und Gefäßchirurgie, Universitätsklinikum Halle, Ernst-Grube-Str. 40, 06097 Halle (Saale) henning.dralle@uk-halle.de

Interessenkonflikt. Der korrespondierende Autor gibt an, dass kein Interessenkonflikt besteht.

\section{Literatur}

1. Dedecjus M, Adamczewski Z, Brzezinski J et al (2010) Real-time, high-resolution ultrasonography of the vocal folds - a prospective pilot study in patients before and after thyroidectomy Langenbecks. Arch Surg 395:859-864 